\title{
RIEMANN SURFACE WITH CYCLIC AUTOMORPHISMS GROUP *
}

\author{
Gustavo Labbé M. \\ Eniversidad de La Serena, Ia Serena, Chile.
}

\begin{abstract}
In this paper. we present the uniformization of $y^{2}=x^{p}-1$, with $\rho>5$ anrl prime. i. e. the only hyperelliptic Rienann surface of genlus y - $\frac{l-1}{2}$, which adnit $Z / 2 p Z$ as automorphism group. This unifumingtion is found by using a fuchsian group which reflects the atrion of $Z:-2 p Z$ and is constructed starting of at triangle group of $\operatorname{sipe}(0, p \cdot p \cdot p)$.

Morower. wescribe completely the action of the automorphism group in homology so that we can deseribe the invariant subvariety for $Z / 2, Z Z$ in $\mathcal{A}_{s}$ (principally polarized abelian varicties of dimension 4). Which is detomined by the ral Abol aplication from $\mathcal{M}_{g}$ in $\mathcal{A}_{g}$.
\end{abstract}

Thi: work was partially supported by DIULS, grant No. 120-2-01 


\section{Introduction}

Fon ${ }_{1}^{1} \mathrm{l}$, and [2;. for to describe the compacts singular locus of $\mathrm{M}_{9}$ (moduli space for compact Rienann surface of genus $y$ ), are important the Rinnane surfares which adnit conformal automorplism grouns isonorphic tu $Z / p Z$, witlı $p$ prinise.

By. 27. it is posible to calculate the number of differont classes for $g$ and $p$ fixen. but it in not known an explicit description and hyperbolic uniformization for these classes.

In this (entext, ure prosent the unifommation of the only hyperelliptic: Rinmann when of genus $g=\frac{p}{2}$. which admit $Z / 2 p Z$ as a antonorphism

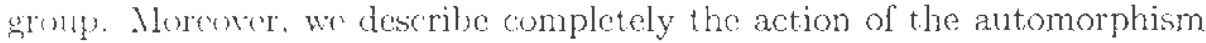
gusup in tomology, so that wo can describe the invariant subvariety for $Z / 2 p Z$ in $\mathcal{A}_{q}$

\section{Riemann Surfaces which Admit $\mathrm{Z} / 2 \mathrm{p} Z$.}

Lot $S$ be the Rirmane surface associated to the algebraic curve $C \subset$

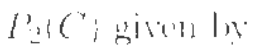

$$
C: z^{p} y^{2}-x^{p}+z^{p}=0 ; \quad p \text { prinue } p>3 .
$$

Then:

(1) $S$ is a compat Riermann surface which is a covering of the Riemann splute ( with $p+1$ branch points.

In fact. consixlen the affine curvo $C_{a}: y^{2}=x^{p}-1$, and put $x_{1}=1$, $x_{2}, \ldots, x_{p}$ the $p^{\text {th }}$-roots of 1 . Then $x_{i}, i=1, \ldots, p$ arc branch points for $\pi: C_{a} \rightarrow C$. lhis may be obtained, writing $y^{2}=\prod_{i=1}^{p}\left(x-x_{i}\right)$ and using the lical parameters $w=x-x_{i}, i=1, \ldots, p$ around $x_{i}$. Thus, $y=\sqrt{u} h(w)$. where $h(0) \neq 0$ and $h$ is analytic: about $w=0$. Hence, $y$ has twe values neal $w=0$, therefore $x_{i}-1, \ldots, p$ are branch points with branching order $b_{\pi}\left(x_{\eta}\right)=1$.

Vuw if wo intersed $G$ with $P_{1}^{\infty}=\{\overline{(x, y, 0)} \mid x, y \in C\}$, we obtain that $x^{\prime}=0$, which implies $x=0$. 
Thuls. $\left(\sqcap / P^{x}=\left\{\overline{(x, y \cdot 0)}=w_{p+1}\right\}\right.$ and $w_{p+1}$ is singular, since

$$
\frac{\partial C}{\partial x}\left(u_{p+1}\right)=\frac{\partial C}{\partial y}\left(u_{p-1}\right)=\frac{\partial C}{\partial z}\left(w_{p+1}\right)=0
$$

$\operatorname{and} b_{\pi}\left(\pi\left(u_{p+1}\right)\right)=1$.

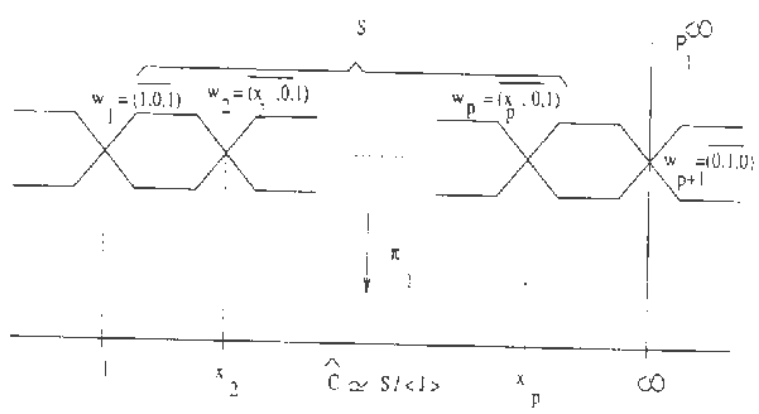

Figure 1:

(2) The gernus of 5 is $y=\frac{p-1}{2}$.

In finct. hy the Rinmann-Hurwitz rolation: $g=n(\gamma-1)+1+\frac{B}{2}$ and fin $n-2 ; z=0: B=p+1$ we have that $g-\frac{p-1}{2}$.

(3) $S$ anduit the hyperelliptic involution $J: S \rightarrow S$ defined by $J \overline{(x, y, z)}=$ $\overline{(r,-y, z)}$ which fixes the $p+1$ points $w_{n}=\overline{\left(x_{i}, 0,1\right)}, i=1, \ldots, p$, $u_{p+1}=\overline{(0.1,0)}$. and therefore $S$ is a hyperelliptic Riemann surface.

(4) Xou we consider the canonical projection $\pi_{1}: S \rightarrow S /\langle J\rangle$.

Since $|\langle J\rangle|=2$, then $\pi_{1}$ is a branched analytic covering of degree two and branched only at the fixed points of $J$. The stability group of wach point is $\langle J\rangle$ and these points are inequivalent under $\langle J\rangle$. 
Thus. the total branch number of $\pi$, is

$B=\sum_{i-1}^{\mu+l} \frac{\mathrm{V}}{\nu_{i}}\left(\nu_{i}-1\right)=p+1 \quad\left(N=2, \nu_{i}=|<J>|=2, i=1, \ldots, p+1\right)$

and by the Riemann-Hurwitz relation, the genus $\gamma$ of $S /<J>$ is zero.

Thenenore the symbol associated to $\pi_{1}: S \rightarrow S /<J>$ is $<\left(\frac{p 1}{2}, 0\right): \underbrace{2,2, \ldots, 2}_{p+1}\rangle$.

(5) Moreover $S$ adnit the autonorphism of order $p, \varphi: S \rightarrow S$ given by $\overline{(x, y+z)}=\overline{(\xi, y, z)}$ where $\xi^{p}=1$ and $\xi \neq 1$.

Tho ramonical projection $\pi_{2}: S \rightarrow S /<\varphi>$ is a branched analytic covering of degree $|\langle\varphi\rangle|=p$ and branched only at the fixes points of $y$ they are $n_{1}=\overline{(0,1, i)} \cdot v_{2}=\overline{(0,-i, 1)} \cdot w_{p+1}=\overline{(0,1,0)}$.

The stability group of each point is $\langle\varphi\rangle$, and thoses points are incunivalent under $\left\langle\varphi>\right.$. Thus, the total branch number of $\pi_{2}$ is givon by

$B=\sum_{i=1}^{3} \frac{V}{\nu_{i}}\left(\nu_{i}-1\right)=3(p-1) \quad\left(N=p, \nu_{i}=|<\varphi>|=p, i=1,2,3\right)$

and by the Riomann-Hurwit relation, the genus $\gamma$ of $S /<J>$ is zero and therefore the symbol associated to $\pi_{2}$ is $\left\langle\left(\frac{p-1}{2}, 0\right) ; p, p, p>\right.$. 
$\$$

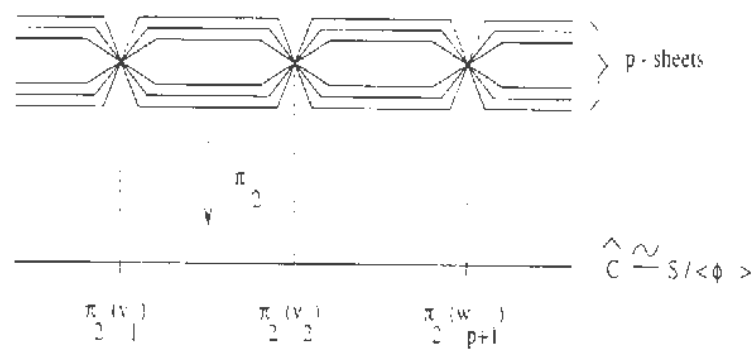

Figure 2 :

(6) Moreover. $S$ admit the automorphism of order $2 p, \psi=\varphi \circ J: S \rightarrow S$ defined by $\varepsilon^{\prime}(x, y, z)=\overline{(\xi x,-y, z)}$ where $\xi^{p}=1$ and $\xi \neq 1$.

Whe "anonis projection $\pi_{3}: S \ldots S /<\measuredangle>$ is a branched analytic

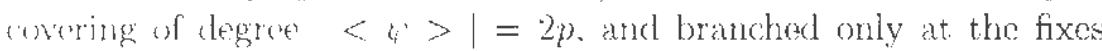
posints of $\langle$ : $\rangle$. they are:

(a) $i^{\prime}=\overline{\langle 0, i, 1)} \cdot i_{2}=\overline{(0,-i .1)}$ with stability group $\langle\varphi\rangle$ and muivalenst under $y$ in $S /\langle\psi\rangle$. Therefore, $v_{1}=|\langle\varphi\rangle|=p$.

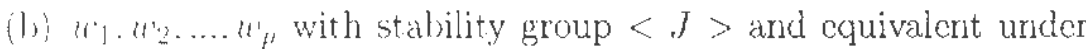
$\rightarrow$ in $S /\langle i\rangle$. Therefore. $\nu_{2}=|\langle J\rangle|=2$.

(c) $u_{p+1}$ with stability group $<\psi>$, therefore $\omega_{3}=2 p$. Thus, in $S /<i_{1}>P=\pi_{3}\left(w_{1}\right) . Q=\pi_{3}\left(w_{1}\right)$ and $O=\pi_{3}\left(w_{p+1}\right)$ arc the brancherl prints of $\pi_{33}$. The total branch number of $\pi_{3}$ is B $.5 p-3$ and by the Riemann-Hurwitz relation, the genus $\gamma$ of $S /\left\langle\psi^{\prime}\right\rangle$ is zoro. and therefore the symbol associated to $\pi_{3}$ is $\left\langle\left(\frac{p-1}{2}, 0\right) ; 2 p \cdot p, 2\right\rangle$. 


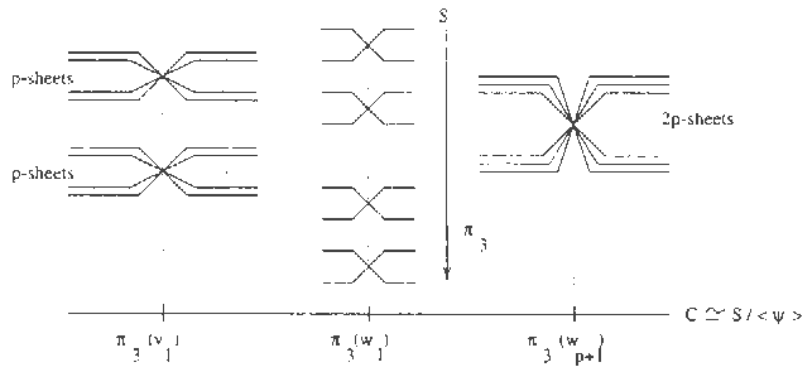

Figure 3:

Throwere. we have the following covering:

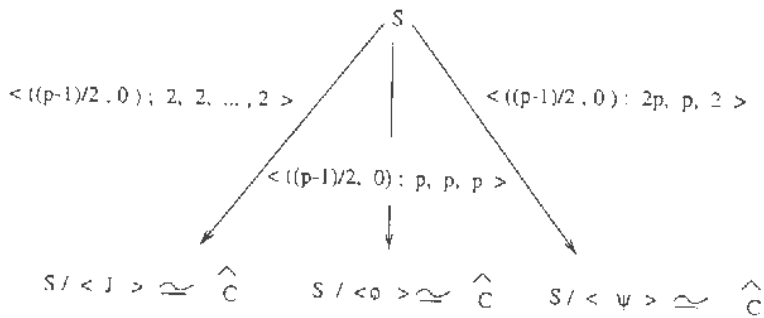

Higure 4: 


\section{Uniformization of $\mathrm{S}$}

By 3 . if $A$ denotes the dise in $C$, then there exists a Fuchsian group $P$ Mafuming the Ritmann sphere $C$ with signature $(0: p, p, p)$ and $T$ has a mesilution $\%=\left\langle a, b ; a^{p}=b^{p}=(a b)^{p}=1\right\rangle$.

Momener, if $: T \rightarrow Z / p Z$ is an epinorphism such that $\Gamma=\operatorname{Ker}(\phi)$ is

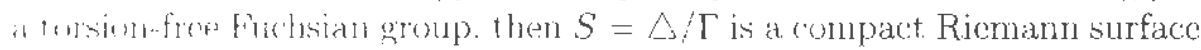

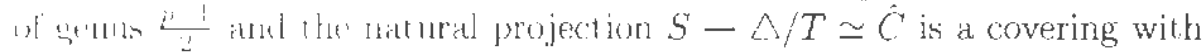
imociand signatule $(0 ; p . p . p)$ and covering group $T / \Gamma \simeq Z / p Z$.

Thus, we have the short exact sequence

$$
0 \longrightarrow \Gamma \longrightarrow T \stackrel{\phi}{\longrightarrow} Z / p Z \longrightarrow 0
$$

and the coverings following:

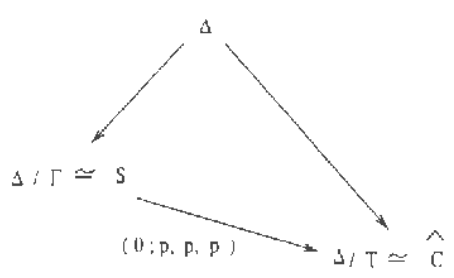

Figure s:

$$
\text { lut } \Phi(\Gamma, T, Z / p Z)=\{\phi: T \rightarrow Z / p Z \mid \phi \text { epimorphism, Ker } \phi \simeq \Gamma\}
$$
where $T=<a, b ; a^{p}=\mid p^{p}=(a b)^{p}=1>$ and $\Gamma$ is a torsion-free group. 
For $\phi_{1} . \phi_{2} \in \Phi(\Gamma . T, Z / p Z)$ we define:

$\phi_{1} \sim \phi_{22}$ if and only if there exists automorphisms $\left\{\begin{array}{ccc}\alpha & : & T \rightarrow T \\ \beta & : & Z / p Z \rightarrow Z / p Z\end{array}\right.$ such that the following diagram is conmutative

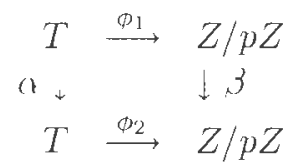

We have that $\sim$ is an equivalence relation and if $\phi_{1} \sim \phi_{2}$ then by [5], $\triangle / \operatorname{Ker}\left(\phi_{1}\right)=S_{1}$ and $\Delta / \operatorname{Ker}\left(\phi_{2}\right)=S_{2}$ are equivalent conformal.

Proposition 1. Let $p$ be a number prime, $p \geq 5$. Then

$$
V(3, p)=\operatorname{Card}(\Phi(\Gamma, T, Z / p Z) / \sim)=\left\{\begin{array}{ccc}
\frac{p+1}{6} & \text { if } & 3 \not(p-1) \\
\frac{p+5}{6} & \text { if } & 3 \mid(p-1)
\end{array}\right.
$$

Thus, there exists $N(3, p)$ Riemann surface $S$ of genus $\frac{p-1}{2}$ which are conformally non-equivalent and such that the covering $S \rightarrow \hat{C}$ has signature $(0: p, p, p)$.

Proof $\mathrm{By}[2]$ and [4]. $N(3, p)$ is the fourth cuefficient of the Taylor series $C_{p}(z)=\sum_{j=0}^{x} \mathrm{~V}(j, p) z^{j}$. where

$G_{p}(z)=\frac{1}{p-1}\left\{\frac{1}{p}\left[(1-z)^{-p+1}+(p-1)(1-z)(1-z)^{-1}\right]+\sum_{l} \phi(l)\left(1-z^{l}\right)^{-\frac{p-1}{l}}\right\}$

and the summation being over $l$ such that $l \neq 1, l \mid(p-1)$ and $\phi(l)$ is the Eulur function.

Therefore, $V(3 \cdot p)=\frac{i_{p}^{\prime \prime \prime}(0)}{6}$. We have two cases:

(a) 1 f $3 \nmid(p-1)$, put $G_{p}(z)=P_{1}(z)+R_{1}(z)$ where

$$
\begin{gathered}
P_{1}(z)=\frac{1}{(p-1) p}(1-z)^{-p+1} \text { and } \\
R_{1}(z)=\frac{1}{p-1}\left\{\frac{1}{p}(p-1)(1-z)\left(1-z^{p}\right)^{-1}+\sum_{l} \phi(l)\left(1-z^{l}\right)^{-\frac{p-1}{l}}\right\},
\end{gathered}
$$

then we obtain $P_{1}^{\prime \prime \prime}(0)=p+1, R_{1}^{\prime \prime \prime}(0)=0$ and therefore $G_{p}^{\prime \prime \prime}(0)=p+1$. 
(1) If 3 $\mid(p-1)$. put $G_{p}(z)=P_{2}(z)+R_{2}(z)$ where

$$
\begin{gathered}
\Gamma_{2}(z)=\frac{1}{p-1}\left\{\frac{1}{p}(1-z)^{-p+1}+\phi(3)\left(1-z^{3}\right)^{-\frac{p-1}{3}}\right\} \text { and } \\
R_{2}(z)=\frac{1}{p-1}\left\{\frac{1}{p}(p-1)(1-z)\left(1-z^{p}\right)^{-1}+\sum_{l . l \leq 3} \phi(l)\left(1-z^{l}\right)^{-\frac{p-1}{l}}\right\},
\end{gathered}
$$

thern we obtain that $P_{2}^{\prime \prime \prime}(0)=p+5 . R^{\prime \prime \prime}(0)=0$ and therefore $G_{p}^{\prime \prime \prime}(0)=$ $p-5$.

\section{Fundamental Polygon.}

Vow we will construct a fundamental polygon $F$ for $T$ using [1], where

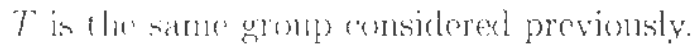

Wo have that $T$ is a tringle group of type $(p, p, p)$ which is presented by $T-<a, b ; a^{i p}-b^{p}=(a b)^{p}=1>$.

Lot $q_{1}, q_{2} \cdot q_{3}$ be fixed points of $a, b$ and $a b$ respectively. If we put $q_{1}$ in the conter of the disc $\triangle$. $q_{2}$ on the positive real axis and $q_{3}$ such that ir: < 11. Wo whtrit a fundamemal polygon $F$ for $T$ as shown in Figure 6 .

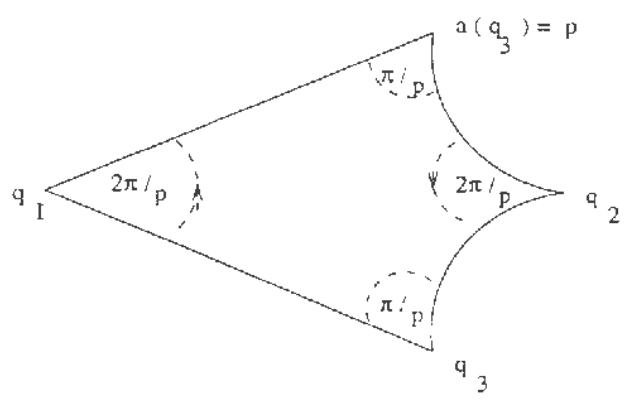

Figure 6: 
Starting from $F$ we build a fundamental region $R$ for $S$, where $S$ has genus ${ }^{2-}$ - $L$. The action of $a$ on $F$ with center in $q_{1}$ repeated $p$-times gives a closed perlygon of 2 p-sides as shown in Figure 7 .

Enumerating the sides of this polygon in the positive sense, the identiticarions of these sides are given by the kernel of the epimorphism $\phi \in$ $\Phi(\Gamma, T, Z / p Z)$.

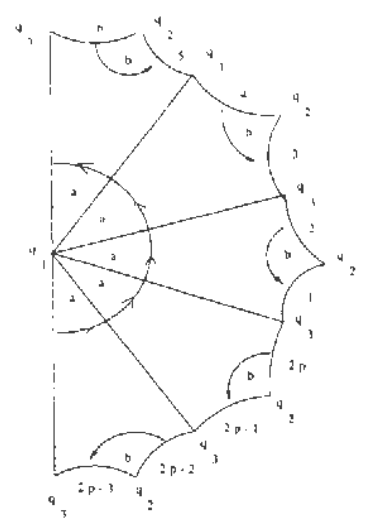

Figure 7 :

Fir wample. the pimorphisn defined by $\phi(a)=1, \phi(b)=\frac{p-1}{2}$, has Kir $=\left\langle a^{2} b^{1}\right\rangle$ which is torsion-free.

We look now at the identifications given by $\operatorname{Ker} \phi$.

If $z$ is a point. which belong to side 1 , then $b^{-1}(z)$ belong to the side 2 and $a \frac{\frac{p-1}{2}}{2} b^{-1}(z)$ belong to the side $2\left(\frac{p-1}{2}\right)+2=p+1$.

If $z$ in at poist belonging to side 2 , then using $\left(a^{\frac{p-1}{2}} b^{-1}\right)^{-1}=b a^{-\frac{p-1}{2}}=$ bxt innterd of $a^{\frac{p-1}{2}} b^{-1}$. wrobtain that $a^{\frac{p+1}{2}}(z)$ bolongs to the side $2\left(\frac{p-1}{2}\right)+$ $2=p+3$ and bo $\frac{\mu+1}{2}(z)$ belongs to the side $(p+3)-1=p+2$.

respoting the same argument, we obtain the identifications:

$$
1 \leftrightarrow(p+1): \quad 2 \leftrightarrow(p+2) ; \cdots: \quad p \leftrightarrow 2 p .
$$


Hence. $R$ is a funclamental region for $S_{\phi}$ and with the identification given above. we obtain that $R / \sim=S_{\phi}$.

Wr note that $S_{\phi}$ is hyperelliptic, since $S_{\phi}$ admit the hyperelliptic involution $J$ given by a rotation of angle $\pi$ around $q_{1}$ in $R$, which fixes the $p$ mid-points of the sides of the polygon and also $q_{1}$.

Therefore, the Riemann surface $S_{\phi}$ is associated with the algebraic curve $z^{p-2} y^{2}-x^{p}+z^{p}=0$.

Moreover. $S_{\phi}$ adnit an automorphism $\varphi$ of order $p$ given by a rotation of angle $\frac{2 \pi}{p}$ around $q_{1}$ in $R$ and admit another automorphism of order $2 p$, $\because=\tau^{\circ} \mathrm{J}$ given by a rotation of angle $\frac{(p+2) \pi}{p}$ around $q_{1}$.

On the other hand, if we build another polygon $R^{\prime}$ of $2 p$-sides centered in $q_{3}$ from $R$ with the identifications given by $\operatorname{Ker} \phi$, then the automorphism of order $2 p$ is a rotation of angle $\frac{\pi}{p}$ around $q_{3}$ of $R^{\prime}$.

\section{Homology Basis of $S_{\phi}$}

Let $R$ be the polygon of $2 p$-sides with the identifications given by $\operatorname{Ker} \phi$, i. e., $j \leftrightarrow(j+p), j=1, \ldots, p$, which uniformizes the hyperelliptic Riemann surface $S=S_{\phi}$ of genus $\frac{p-1}{2}$.

For will obtain an homology basis of $S_{\phi}$, we consider $U=\left\{q_{2}, q_{3}\right\}$ and the short exact sequence in homology:

$$
0 \longrightarrow H_{1}(S) \longrightarrow H_{1}(S, U) \stackrel{\diamond}{\longrightarrow} H_{0}(U) \longrightarrow H_{0}(S) \longrightarrow 0
$$

where $H_{1}(S)$ is the first homology group of $S . H_{1}(S, U)$ is the first relative homolugy group and $\delta$ is defined by $\delta(\gamma)=\gamma(1)-\gamma(0)$.

Therefore. the seculence in homology is

$$
0 \longrightarrow H_{1}(S) \longrightarrow H_{1}(S, U) \longrightarrow Z \oplus Z \longrightarrow Z \longrightarrow 0,
$$

and the homology basis for $S$ is given by Ker $\delta$.

If we identify the sides $1,2 \ldots \ldots p$ by the polygon $R$ with the paths $3_{1}, 3_{2} \ldots, \xi_{p}$ with the positive orientation, then $1+p, 2+p, \ldots, 2 p$ correspond respectively to $\beta_{1}^{-1}, \beta_{2}^{-1}, \ldots, \beta_{p}^{-1}$ and $\beta_{1}, \beta_{2}, \ldots, \beta_{p}$ are generators for $H_{1}(S, U)$. 
Let $: \in$ korn be then $\gamma=\sum_{t=1}^{p} m_{n}, 3_{2}$. with $m_{i} \in Z$ and

$$
\phi(\gamma)=q_{3}\left(\sum_{j=1}^{p}(-1)^{j} m_{j}\right)+q_{2}\left(\sum_{j=1}^{p}(-1)^{j+1} m_{j}=0\right.
$$

hence, $m_{1}=\sum_{j-2}^{p}(-1)^{j} m_{j}$ and we obtain an homology basis for $S,\left\{\alpha_{i}\right\}_{i=1}^{p-1}$ where $n_{i}, b_{i}+f_{i+1}$. with $i=1, \ldots, p+1$ as shown in Figure 8 .

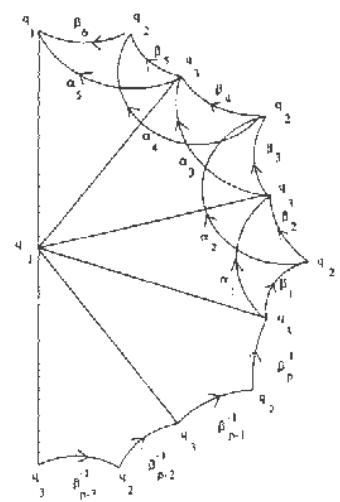

Figure 8:

Let $\psi^{*}=\varphi_{0}, S \longrightarrow S$ be the automorphism of order $2 p$, then " : $H_{1}(S) \longrightarrow H_{1}(S)$ in lerm of the basis $\left\{a_{i}\right\}_{i=1}^{p}$ is given by

$$
\left\{\begin{aligned}
\psi\left(\alpha_{i}\right) & =-\alpha_{i+2}, \quad i=1, \ldots, p-3 \\
\psi\left(\alpha_{p-2}\right) & =\sum_{j=1}^{p-1}(-1)^{j+1} \alpha_{j} \\
\psi\left(\alpha_{p-1}\right) & =\alpha_{1}
\end{aligned}\right.
$$

and therefore is represented for the matrix 


$$
A_{2 p}=\left(\begin{array}{rrrrrrr}
0 & 0 & 0 & \cdots & 0 & 1 & 1 \\
0 & 0 & 0 & \cdots & 0 & -1 & 0 \\
-1 & 0 & 0 & \cdots & 0 & 1 & 0 \\
0 & -1 & 0 & \cdots & 0 & -1 & 0 \\
0 & 0 & -1 & \cdots & 0 & 1 & 0 \\
\vdots & \vdots & \vdots & \vdots & \vdots & \vdots & \vdots \\
0 & 0 & 0 & \cdots & -1 & -1 & 0
\end{array}\right)
$$

The matrix $A_{2 p}$ satisfies: $A_{2 p}^{p}=-I$ and $A_{2 p}^{2 p}=I$.

\section{Example}

Let $S$ be the Riemann surface associated to the algebraic curve

$$
C: \quad z^{11} y^{2}-x^{13}+z^{13}=0 .
$$

Then, $S$ is a Riemann surface of genus six and $S$ admit the hyperelliptic involution $J$, hence is a hyperelliptic Riemann surface.

Moreover, $S$ admit another automorphism $\varphi$ of order thirteen and the (anonical projection $\pi: S \rightarrow S /\langle\varphi\rangle$ is a covering with signature $<(6.0): 13.13 .13\rangle$.

Also, $S$ admit another automorphism $\psi=\varphi \circ J$ of order twenty-six and the canonical projection $\pi: S \rightarrow S /\langle\psi\rangle$ is a covering with signature $<(6.0): 26,13.2\rangle$.

Lut $T$ be a triangle group of type $(13,13,13)$ presented by

$$
T=\left\langle a, b ; a^{13}=b^{13}=(a b)^{13}=1>.\right.
$$

Then, given an epimorphism $\phi: T \rightarrow Z / 13 Z$ such that $\Gamma=\operatorname{Ker} \phi$ is torsionfree. $S=\triangle / \Gamma$ is a Riernann surface of genus six and the canonical projection $S \rightarrow \triangle / T$ is a covering with signature $(0 ; 13,13,13)$ and covering group $T / \Gamma \simeq Z / 13 Z$.

Then. by lemma previous, there exists three Riemann surfaces $S$ of genus six which signature $(0,13,13,13)$, one of these is the hyperclliptic Rienanm surface associated to algebraic curve $z^{11} y^{2}-x^{13}+z^{13}=0$. 
These surfaces are given by the three different elasses of epimorphisms c) $T \rightarrow Z / 13 Z$ with $\mathrm{Ker} \phi$ torsion-free and $S=\triangle / \mathrm{Ker} \phi$.

Now, the fundantental polygon $F$ for $T$ is shown in the following Figure, where $q_{1}$ is the fixed point of $a, q_{2}$ is the fixed point of $b$ and $q_{3}$ is the fixed point of als.

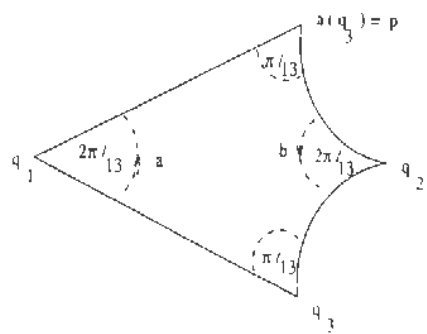

whore: of is the tianed poral of $\mathrm{a}_{\mathrm{r}}$

$q_{2}$ the lixed poin of $b$.

q is the fixed fom of ah

Figure 9:

Starting with $F$, we build a fundamental region $R$ for $S$ as shown in Figlite 10 .

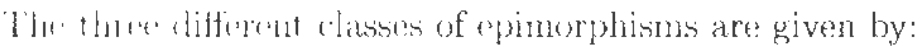

(i) $\phi_{1}(a)=1: \phi_{1}(b)=b, \operatorname{Kor} \phi_{1}=\left\langle a^{6} b^{1}\right\rangle$ that gives the identifications

$$
1 \leftrightarrow 14: \quad 2 \leftrightarrow 15 ; \quad 3 \leftrightarrow-16: \quad \cdots \quad ; 13 \leftrightarrow 26 .
$$

I'herfere. $S_{!}=\Delta / K_{e r} \phi_{1}$ is the hyleselliptic: Riemann surface.

(ii) $y_{2}(a) !, \phi_{2}(b)=2$. Kor $\phi 2=<a^{2} b^{2}>$ that gives the identifications

$$
1 \rightarrow 6 ; \quad 2+2: 3 ; \quad 3 \leftrightarrow 8 ; \quad 1,25 ; \quad 5 \leftrightarrow 10 ; \quad 7 \leftrightarrow 12 ; \quad 9 \leftrightarrow 14 ;
$$

$$
11 \rightarrow 16 ; \quad 13 \leftrightarrow 18 ; \quad 15 \leftrightarrow 20 ; \quad 17 \leftrightarrow 22 ; \quad 19 \leftrightarrow 24 ; \quad 21 \leftrightarrow 26 .
$$


(iii) $o_{3}(a)=1, Q_{3}(b)=3$ Ker $\phi_{3}=\left\langle a^{3} b^{-1}>\right.$ that gives the identifications $1 \hookleftarrow 8: \quad 2 \hookleftarrow 21: \quad 3 \leftrightarrow 10 ; \quad 4 \leftrightarrow 23 ; \quad 5 \leftrightarrow 12 ; \quad 6 \leftrightarrow 25 ; \quad 7 \leftrightarrow 14 ;$ $9 \leftrightarrow 16: \quad 11 \leftrightarrow 18 ; \quad 13 \leftrightarrow 20 ; \quad 15 \leftrightarrow 22 ; \quad 17 \leftrightarrow 24 ; \quad 19 \leftrightarrow 26$.

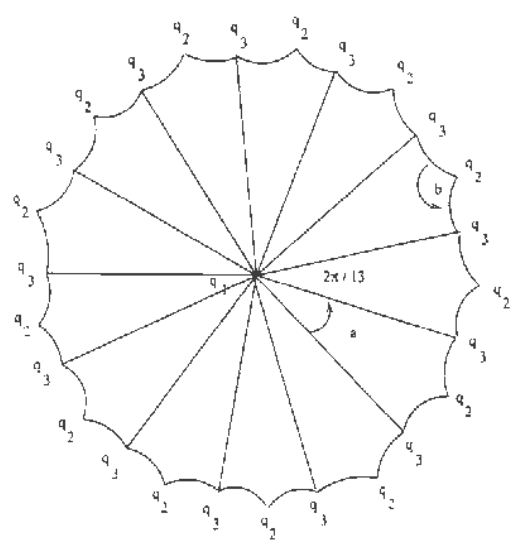

Figure 10:

An homology basis for the hyperelliptic Riemann surface is given by the paths $\left\{\alpha_{q}\right\}_{i=1}^{12}$, as shown in Figure 11.

The hyperelliptic involution $J$ is given by a rotation of angle $\pi$ around $q_{1}$. the antomorphisin $\varphi$ of order thirteen is given by a rotation of angle $\frac{2 \pi}{13}$ around $q_{1}$ and the automorphism $\psi=\varphi^{\circ} J$ of order twenty-six is given by a ritation of angle $\frac{15 \pi}{13}$ around $q_{1}$. 


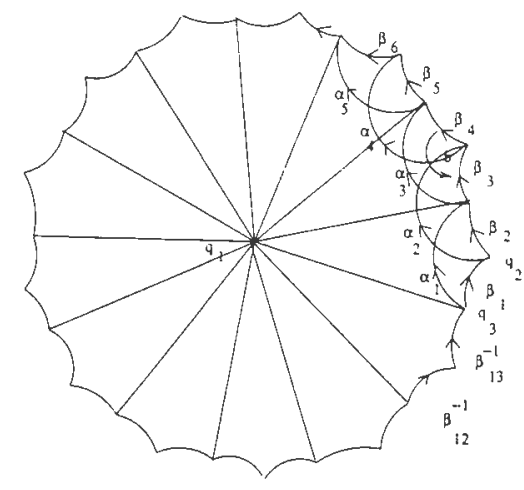

Figure 11:

The automorphism $\psi$ in homology in the basis $\left\{\alpha_{i}\right\}_{i=1}^{12}$ is given by

$$
A_{26}=\left(\begin{array}{rrrrrrrrrrrr}
0 & 0 & 0 & 0 & 0 & 0 & 0 & 0 & 0 & 0 & 1 & 1 \\
0 & 0 & 0 & 0 & 0 & 0 & 0 & 0 & 0 & 0 & -1 & 0 \\
-1 & 0 & 0 & 0 & 0 & 0 & 0 & 0 & 0 & 0 & 1 & 0 \\
0 & -1 & 0 & 0 & 0 & 0 & 0 & 0 & 0 & 0 & -1 & 0 \\
0 & 0 & -1 & 0 & 0 & 0 & 0 & 0 & 0 & 0 & 1 & 0 \\
0 & 0 & 0 & -1 & 0 & 0 & 0 & 0 & 0 & 0 & -1 & 0 \\
0 & 0 & 0 & 0 & -1 & 0 & 0 & 0 & 0 & 0 & 1 & 0 \\
0 & 0 & 0 & 0 & 0 & -1 & 0 & 0 & 0 & 0 & -1 & 0 \\
0 & 0 & 0 & 0 & 0 & 0 & -1 & 0 & 0 & 0 & 1 & 0 \\
0 & 0 & 0 & 0 & 0 & 0 & 0 & -1 & 0 & 0 & -1 & 0 \\
0 & 0 & 0 & 0 & 0 & 0 & 0 & 0 & -1 & 0 & 1 & 0 \\
0 & 0 & 0 & 0 & 0 & 0 & 0 & 0 & 0 & -1 & -1 & 0
\end{array}\right)
$$




\section{lieferences}

If Comalba M.. On the Locus of Curves weth Automorphisms. Annali di Mat. Pural et Appl., 149, (1987), 135151.

'2. Harvey. W. J., On branch loci in Teichmuller space. Transaction A. H. S. (1971)

3 Gonzálo V. Rira G. \& Rodrí guez. R. On Riemann Matrices and Iniformazations lof Riemann Surfaces with Automorphism. Complex Geometry Semiriar, Vol II, 1986, U.T.F.S.M.

[4] Lloyd, E. K., Riemann Surface Transformation groups. Ph. D. Thesis. Birningham. 1967.

5) Macbenth. A. M.. Procieding of the Summer School in Geometry and Topology. Quen's 's College. Dundere. 1961.

Rerevived : 10 de Einero 1996.

\section{Gustavo Labbé M}

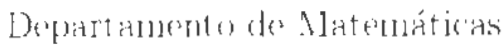

Fatcultad de Cierretas

Universidad de: La Serena

Casilla 559

Lat Sercila

Chilc 